\title{
Pravastatin attenuates allergic airway inflammation by suppressing antigen sensitisation, interleukin 17 production and antigen presentation in the lung
}

\author{
M Imamura, ${ }^{1}$ K Okunishi, ${ }^{1}$ H Ohtsu, ${ }^{2}$ K Nakagome, ${ }^{1}$ H Harada, ${ }^{1}$ R Tanaka, ${ }^{1}$ \\ K Yamamoto, ${ }^{1}$ M Dohi'
}

- Supplementary data are published online only at http:// thorax.bmj.com/content/vol64/ issue1

${ }^{1}$ Department of Allergy and Rheumatology, Graduate School of Medicine, University of Tokyo, Tokyo, Japan; ${ }^{2}$ Department of Clinical Trial Data Management, Graduate School of Medicine, University of Tokyo, Tokyo, Japan

Correspondence to: Dr M Dohi, University of Tokyo Department of Allergy and Rheumatology, Graduate School of Medicine, 7-3-1 Hongo,

Bunkyo-ku, Tokyo 113-8655,

Japan; mdohi-tky@umin.ac.jp

Received 11 December 2007 Accepted 22 August 2008 Published Online First

3 October 2008

\begin{abstract}
Background: Statins are widely used to treat hyperlipidaemia. Their immunosuppressive effect has recently been confirmed in various immune mediated disease models. However, relatively few studies have been conducted on allergic inflammation, so the precise mechanisms of their actions against allergies have not been fully clarified. On the other hand, the role of interleukin (IL)17 in immune responses has been recently highlighted, but whether statins affect IL17 production has not been well studied. The effect of pravastatin on allergic airway inflammation in a mouse model was examined to elucidate the mechanism of action, focusing on its effect on IL17 production.
\end{abstract}

Methods: BALB/c mice were immunised with ovalbumin (OVA) and then challenged with OVA aerosol. Pravastatin was delivered by intraperitoneal injection during either sensitisation or the challenge.

Results: When delivered during systemic sensitisation, pravastatin suppressed OVA induced proliferation and production of Th2 type cytokines such as IL5 in spleen cells ex vivo and in vitro. IL17 production was also suppressed. Furthermore, pavastatin delivered during the inhalation of OVA attenuated eosinophilic airway inflammation, OVA specific IgE production in serum and OVA induced IL17 production in the thoracic lymph node. We also found that pravastatin attenuated the antigen presenting capacity of $\mathrm{CD} 11 \mathrm{c}^{+}$cells obtained from the OVA challenged lung.

Conclusion: Pravastatin suppresses the systemic sensitisation to allergen with downregulation of IL17 production. It also suppresses an ongoing immune response in the airway partly by suppressing antigen presentation in the lung. Therefore, statins could be a novel therapeutic option for treatment of asthma.

Statins are inhibitors of the 3-hydroxy-3-methylglutaryl coenzyme A (HMG-CoA) reductase, and have been widely prescribed to treat hyperlipidaemia. Large clinical trials have demonstrated that statins can be used for primary and secondary prevention of cardiovascular diseases. ${ }^{1}$

In addition to their conventional lipid lowering properties, statins are now recognised as possessing a variety of immunomodulatory effects. ${ }^{2}$ Statins downregulate various functions of antigen presenting cells (APCs), such as dendritic cells $s^{3-5}$ and $B$ cells. ${ }^{6}$ Furthermore, statins promote in vitro $\mathrm{T}$ helper (Th) 2 polarisation from naïve $\mathrm{CD} 4^{+} \mathrm{T}$ cells via suppression of Th1 development. ${ }^{7}$ In animal models of Th1 biased autoimmune disorders such as experimental autoimmune encephalomyelitis ${ }^{58}$ and collagen induced arthritis, ${ }^{9}$ statin attenuates disease severity with a shift from a Th1-type to Th2-type cytokine profile.

Allergic bronchial asthma is a Th2 mediated disease, and statins will exaggerate allergic inflammatory responses in the airway. However, in several reports, statins suppressed Th2 type airway inflammation. ${ }^{10-13}$ Simvastatin and pravastatin reduced production of Th2-type cytokines, thus attenuating allergic airway inflammation. ${ }^{10-12}$ However, the precise mechanisms of action have not yet been fully clarified. ${ }^{13}$ Further details should be clarified for clinical application.

A significant role for interleukin (IL)17 producing $\mathrm{CD}^{+} \mathrm{T}$ cells (Th17 cells) has recently been highlighted. ${ }^{14-16}$ Generally, IL17 is critical to host defence against various infections. ${ }^{17}{ }^{18}$ Furthermore, IL17 affects various immune responses. Many immunological processes have recently been reevaluated in the context of Th17 and regulatory T cells. ${ }^{14}$ For example, both the encephalomyelitis model and collagen induced arthritis model have been considered Th17 mediated models rather than conventional Th1 models. ${ }^{14} 1920$ IL17 also plays an important role in neutrophil dominated inflammatory responses in the lung. ${ }^{21}$ Recently, the role of IL17 in asthma has also been intensively studied. ${ }^{22-27}$ For example, elevated sputum IL17 concentrations correlate with clinical severity. ${ }^{22}$ The role of IL17 in an animal model of allergic airway inflammation has been investigated in several studies. ${ }^{23-25}$ In one report, IL17 was essential for sensitisation to the antigen whereas it attenuated airway inflammation in the effector phase. ${ }^{23}$ At present, the precise role of IL17 in allergic airway inflammation is not fully clear. In addition, whether statins affect IL17 mediated responses has not been well studied.

This study was performed to examine the hypothesis that pravastatin attenuates allergic airway inflammation through suppression of IL17 production. We conducted research in vitro and in vivo using an animal model. We found that pravastatin suppressed systemic sensitisation to the antigen with downregulation of IL17 production, and thus suppressed antigen induced eosinophilic airway inflammation, partly by regulating antigen presentation in the lung.

\section{METHODS \\ Mice}

Male BALB/c mice, 7 weeks of age, were obtained from Charles River Japan (Kanagawa, Japan). Male $\mathrm{BALB} / \mathrm{c} \mathrm{T}$ cell receptor transgenic DO11.10 mice, 
aged 6-8 weeks, were purchased from The Jackson Laboratory (Bar Harbor, Maine, USA). They were maintained under conventional animal housing conditions in a specific pathogen free setting. All experiments were approved and performed according to the guidelines of the University of Tokyo.

\section{Study protocol in vivo}

$\mathrm{BALB} / \mathrm{c}$ mice were immunised with an intraperitoneal injection of $2 \mu \mathrm{g}$ of ovalbumin (OVA; Sigma-Aldrich, St Louis, Missouri, USA)/2 mg of aluminium hydroxide (alum) on days 0 and 11. Control mice received saline instead of the OVA/alum solution. The animals were challenged with an aerosolised solution of $3 \%$ w/v OVA in phosphate buffered saline (PBS) for $10 \mathrm{~min}$ from day 18 to day 20. Pravastatin $(10 \mathrm{mg} / \mathrm{kg}$, kindly provided by Sankyo Co, Tokyo, Japan) dissolved in $0.5 \mathrm{ml}$ of saline, or saline alone, was given by intraperitoneal injection 30 min before inhalation of OVA on days 18, 19 and 20. Control mice inhaled PBS on days 18-20. On day 21, samples of serum, bronchoalveolar lavage fluid (BALF) and lungs were obtained. The lungs were cut out and used for analyses of antigen presentation. In another experiment, left lungs were homogenised in $1.0 \mathrm{ml}$ of PBS containing $0.5 \%$ Triton X-100 and complete protease inhibitor cocktail (Roche, Basel, Switzerland). The lung homogenates were cleared of debris and cells by centrifugation at $10000 \times \mathrm{g}$ for $10 \mathrm{~min}$. Thoracic lymph nodes were also obtained and used for ex vivo analyses. Cell counts and cell differentials of BALF were determined as previously reported. ${ }^{28-30}$ Cytokine concentrations in BALF, lung homogenates and immunoglobulin levels in sera were measured by enzyme linked immunosorbent assay (ELISA). The numbers of animals were six in the non-sensitised group, and 8-14 in the saline treated or pravastatin treated sensitised groups, respectively. We conducted the experiment more than four times and confirmed the reliability of the data.

\section{Quantification of cytokines, cell proliferation and cholesterol}

Cytokine concentrations in BALF, serum and cell culture supernatants were determined by ELISA ${ }^{31}$ (see methods in the online data supplement).

\section{Preparation of single cell suspensions of spleen and lymph node cells}

Spleens were collected and incubated at $37^{\circ} \mathrm{C}$ for 15 min after treatment with $0.1 \%(\mathrm{w} / \mathrm{v})$ collagenase (Sigma-Aldrich)/complete Dulbecco's modified Eagle's medium (DMEM) solution and then minced. Lymph node cells were collected and minced and then incubated at $37^{\circ} \mathrm{C}$ for $30 \mathrm{~min}$ with $0.033 \%$ collagenase/complete DMEM solution. Single cell suspensions were prepared with a cell strainer. Red blood cells were removed by hypotonic lysis. After two washes, the cells were used for experiments. Complete DMEM was used for incubation throughout the study. ${ }^{28-30}$

\section{Response of spleen and lymph node cells to OVA}

Spleen or lymph node cells $\left(2.5 \times 10^{6}\right.$ cells $\left./ \mathrm{ml}\right)$ were cultured in a 96 well, flat bottomed microtitre assay plate with OVA $(100 \mu \mathrm{g} / \mathrm{ml})$ in an incubator $\left(37^{\circ} \mathrm{C}, 5 \% \mathrm{CO}_{2}, 90 \%\right.$ humidity). Cell proliferation was measured on day 3 . On day 4 , cytokine production was measured by ELISA.

\section{Animal preparation for ex vivo analyses}

$\mathrm{BALB} / \mathrm{c}$ mice were immunised with OVA/alum on days 0 and 11. On days $11-17$, some mice received pravastatin $(10 \mathrm{mg} / \mathrm{kg})$ dissolved in $0.5 \mathrm{ml}$ of saline or saline alone by intraperitoneal injection. Control mice received saline on days 0 and 11. On day 18, spleens were collected and cell proliferation and cytokine production were measured.

\section{Effects of pravastatin on antigen presentation in the lung}

On day 21, CD11 $\mathrm{c}^{+}$antigen presenting cells in the lungs of mice were positively selected, as reported previously. ${ }^{28-30}$ In brief, lung tissues were minced and then treated with $0.033 \%(\mathrm{w} / \mathrm{v})$ collagenase/complete DMEM solution for $30 \mathrm{~min}$. Single cell suspensions of the tissues were obtained, and CD11c ${ }^{+}$cells were positively selected using MACS CD11c microbeads (Myltenyi Biotec, Auburn, California, USA). The populations of cells selected from lung tissues were routinely $\sim 70 \% \mathrm{CD} 11 \mathrm{c}^{+}$, and there was no significant difference in the purity of $\mathrm{CD} 11 \mathrm{c}^{+}$cells between the groups of mice. Preliminary experiments demonstrated that almost $70 \%$ of the $\mathrm{CD} 11 \mathrm{c}^{+}$cells expressed the major histocompatibility complex class II molecule I-A/I-E. Some CD11 $\mathrm{c}^{+}$cells co-expressed F4/80 or Gr-1 (1-2\%), an indicator of macrophages and granulocytes, respectively. Lung CD11 $\mathrm{c}^{+}$cells had negligible expression of CD4, CD8 $\alpha$ and CD19 (data not shown). These results suggest that although lung CD11 $\mathrm{c}^{+}$cells contain some subsets, the main population of $\mathrm{CD}_{11 \mathrm{c}^{+}}$cells were dendritic cells. These $\mathrm{CD}_{11 \mathrm{c}^{+}}$cells were incubated with mitomycin C $\left(10 \mu \mathrm{g} / \mathrm{ml}\right.$; Sigma-Aldrich) for $35 \mathrm{~min}$ at $37^{\circ} \mathrm{C}$ in a humidified atmosphere containing $5 \% \mathrm{CO}_{2}$ and then washed four times with PBS. In addition, $\mathrm{CD}^{+} \mathrm{T}$ cells were obtained from spleen cells of the DO11.10 mice using anti-mouse CD4 colloidal superparamagnetic microbeads (Myltenyi Biotec), as previously reported. ${ }^{28-30}$ The purity of $\mathrm{CD}^{+}$cells, confirmed by flow cytometry, was $>95 \%$. For measuring antigen presenting capacity, lung $\mathrm{CD}_{11 \mathrm{c}^{+}}$cells $\left(0.25 \times 10^{4}, \quad 0.8 \times 10^{4}\right.$, and $2.5 \times 10^{4}$ cells $/ \mathrm{ml}$ ) obtained from each group of mice were cocultured with $\mathrm{CD}^{+} \mathrm{T}$ cells $\left(2.5 \times 10^{5}\right.$ cells $\left./ \mathrm{ml}\right)$ selected from the spleens of DO 11.10 mice. After a 2 day coculture, proliferation of $\mathrm{CD}^{+} \mathrm{T}$ cells was measured.

\section{Statistical analysis}

We applied data from at least three sets of samples from individual animals to statistical analysis, while we did not apply data from individual culture wells. Values are expressed as mean (SD) or individual points. The Komolgorov-Smirnov test was used to check the normal distribution of data. For all variables, the numbers of lymphocytes and IL13 levels in BALF rejected normality. Differences in variables between the groups were determined by the Student's t test or the Mann-Whitney U test for variables with or without normal distribution, respectively. Values of $\mathrm{p}<0.05$ were considered significant.

\section{RESULTS}

Pravastatin decreased eosinophilic inflammation in the lung and suppressed total $\lg E$, OVA specific $\lg E$ and $\lg G 1$ levels in serum Treatment with pravastatin significantly suppressed eosinophilic airway inflammation induced in OVA treated mice (fig 1A, 1B). We conducted this experiment repeatedly, and the numbers of eosinophils in the BALF of mice treated by pravastatin were $30-60 \%$ of those of OVA treated mice. Concentrations of IL13 and transforming growth factor $\beta$ in BALF were also significantly suppressed (fig 1C, 1D). Although the IL17 content of the lung increased in the OVA treated mice compared with the saline treated control mice, it did not seem to be affected by pravastatin (fig 1E). Pravastatin treatment significantly reduced serum total IgE, OVA specific IgE and OVA specific IgG1 levels, while no significant difference was observed in the total IgG and 
A
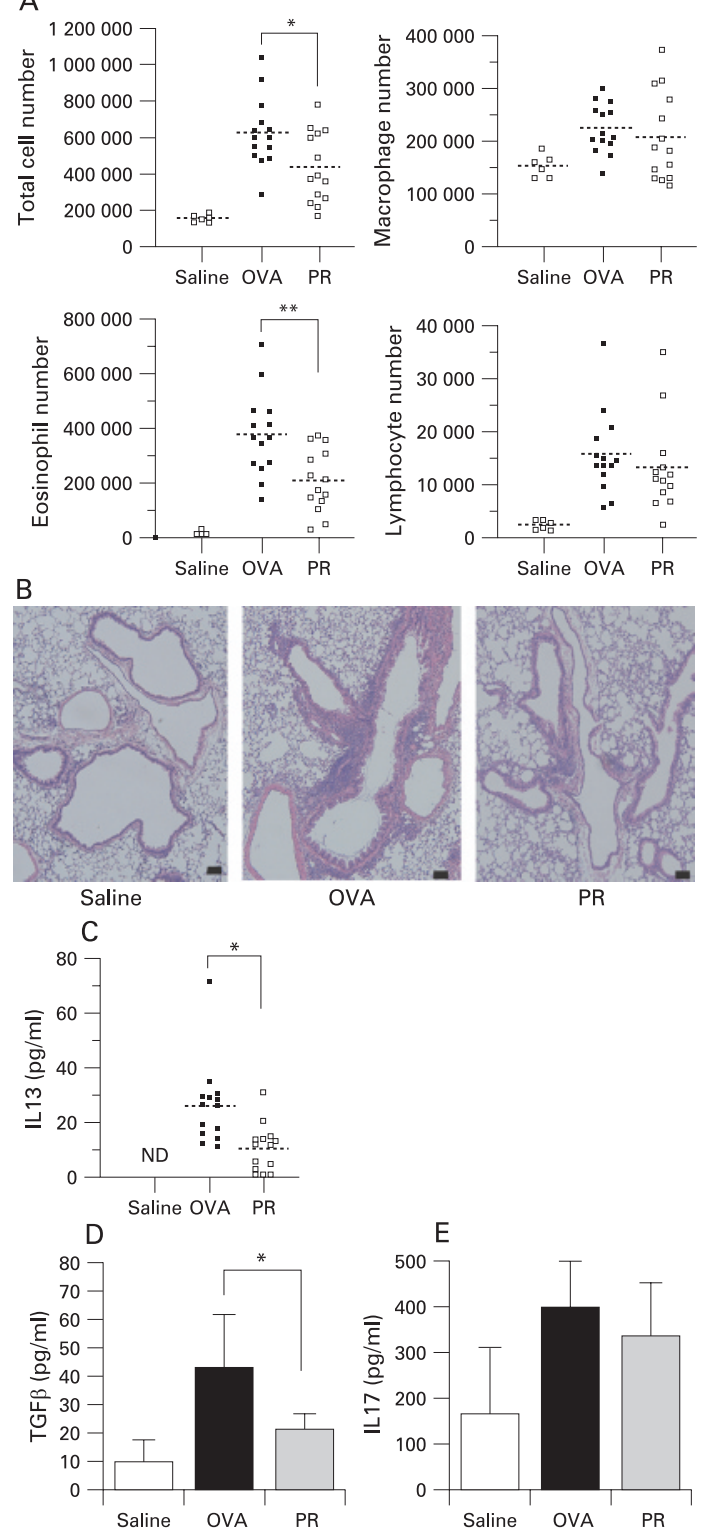

Figure 1 Treatment with pravastatin during ovalbumin (OVA) challenge significantly attenuated the development of allergic airway inflammation. Mice were sensitised with OVA on days 0 and 11. Then, mice were challenged with OVA aerosol on days 18-20. On days 18-20, they received intraperitoneal injections of pravastatin (PR) or control vehicle (OVA) 30 min before the challenge. Control mice received saline injections on days 0 and 11, and phosphate buffered saline (PBS) aerosol challenges on days 18-20 (saline). On day 21, bronchoalveolar lavage fluid (BALF) and lung tissues were obtained. (A) Cell differentials in BALF. Each point represents an individual mouse. (B) Haematoxylineosin staining of lung tissues (scale bar $100 \mu \mathrm{m}$ ). (C) Interleukin (IL)13 concentration in BALF. Each point represents an individual mouse. (D, E) Transforming growth factor $\beta$ (TGF $\beta$ ) in BALF (D) and IL17 in the lung homogenate (E). Data are expressed as mean (SD). Data were obtained from six animals (saline) and 8-14 animals (OVA, PR) per group of mice. ${ }^{*} \mathrm{p}<0.05$; ${ }^{* *} \mathrm{p}<0.01$, between OVA sensitised/challenged mice (OVA group) and OVA sensitised/challenged and pravastatin treated mice (PR group). Data are representative of more than four independent experiments. ND, not detectable.

OVA specific IgG levels (fig 2A-E). Pravastatin did not affect total serum cholesterol levels (saline treated mice 110.4 (12.4) mg/dl; OVA treated mice 102.6 (7.0) mg/dl; pravastatin
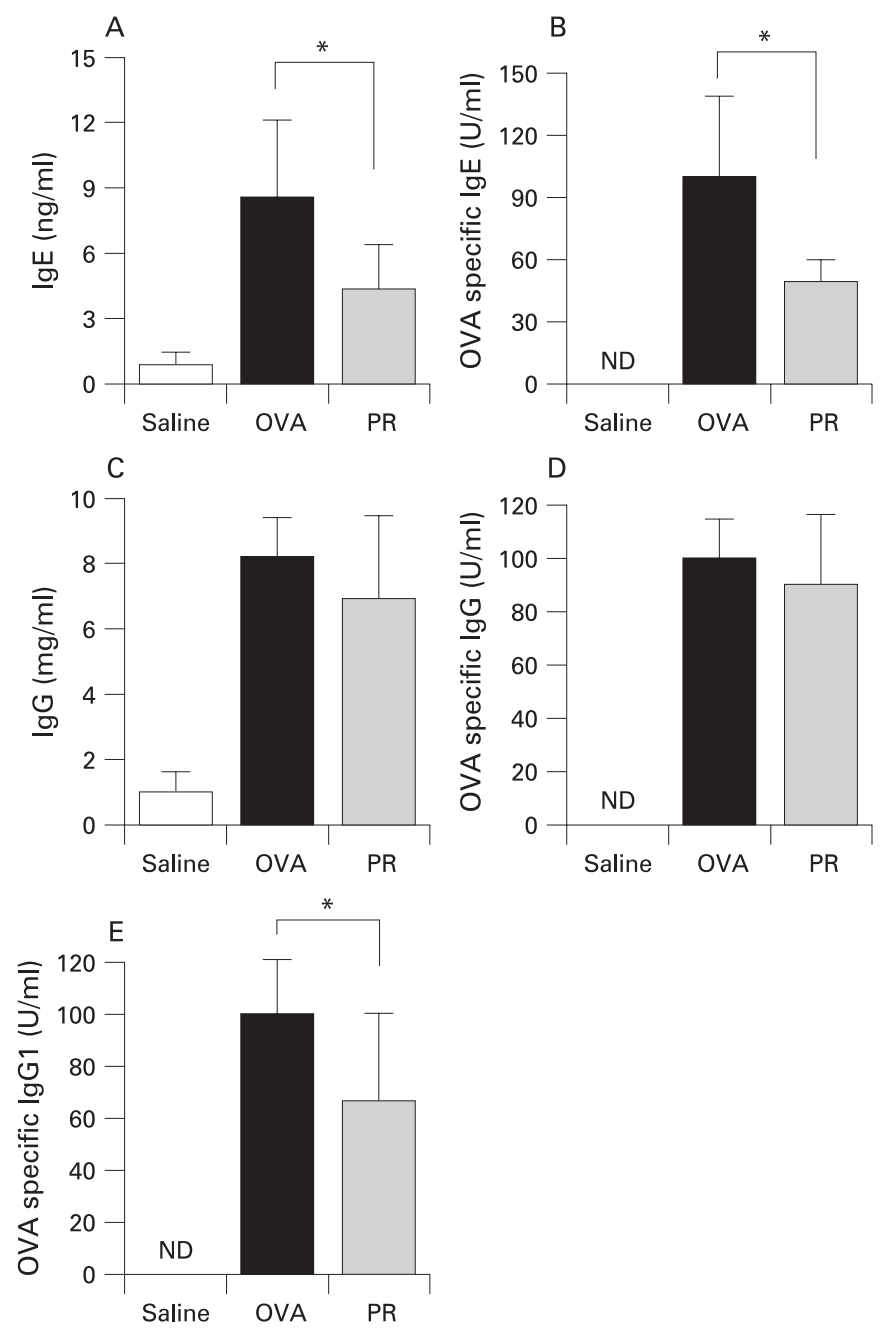

Figure 2 Treatment with pravastatin during ovalbumin (OVA) challenge significantly attenuated serum $\lg \mathrm{E}$ and $\lg \mathrm{G} 1$ production. Mice were subjected to analyses as described in fig 1. On day 21, serum was obtained. Total $\lg E(A)$, OVA specific $\lg E(B)$, total $\lg G(C)$, OVA specific $\lg \mathrm{G}(\mathrm{D})$ and OVA specific $\lg \mathrm{G} 1$ (E) in the serum. Data are expressed as mean (SD). Data were obtained from six animals (saline), and 8-14 animals (OVA group, pravastatin (PR) group) per group of mice. ${ }^{*} p<0.05$ difference between OVA and PR group. Data are representative of more than four independent experiments. ND, not detectable.

treated mice $106.3(11.0) \mathrm{mg} / \mathrm{dl}$ ). Therefore, the suppressive effect was independent of serum cholesterol levels.

\section{Treatment with pravastatin during systemic sensitisation} suppresses the antigen induced immune response of spleen cells Cell proliferation and production of IL5, IL10 and IFN- $\gamma$ by splenocytes obtained from OVA treated mice were increased compared with saline treated mice (fig $3 \mathrm{~A}-\mathrm{D}$ ). IL17 production was also increased in OVA treated mice, and pravastatin seemed to suppress IL17 production (fig 3E). Furthermore, pravastatin treatment tended to reduce these responses to OVA except the production of IFN $\gamma$ (fig $3 \mathrm{~A}-\mathrm{E}$ ). In another experiment, pravastatin treatment in vitro tended to suppress cell proliferation and production of IL5, IL17 and IL10 in a dose dependent manner (see supplementary fig 1 with method online). Hence treatment with pravastatin during a second systemic sensitisation attenuated the Th2-type antigen induced immune response with suppression of IL17 production. 

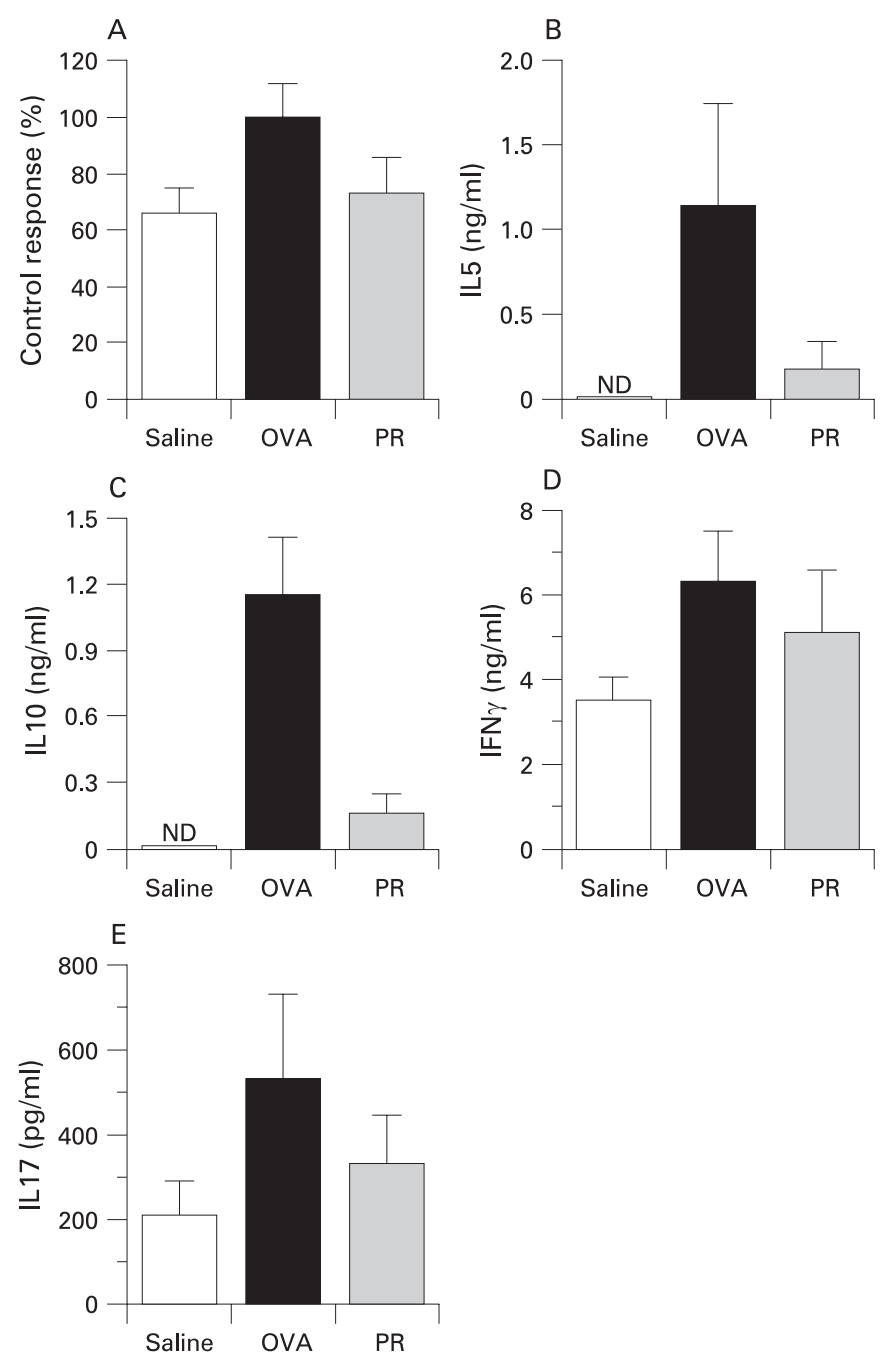

Figure 3 Treatment with pravastatin (PR) during sensitisation in vivo decreases spleen cell responses to ovalbumin (OVA) restimulation. Mice received OVA/alum or saline injections on days 0 and 11. The OVA sensitised mice received intraperitoneal injections of pravastatin or saline (OVA) on days 11-17. Spleen cells obtained on day 18 were restimulated in vitro with OVA. (A) Cell proliferation was measured after 3 days of incubation. Data are expressed as a percentage of the response compared with that of spleen cells from OVA mice. (B-D) Production of interleukin (IL)5 (B), IL10 (C), IFN $\gamma$ (D) and IL17 (E) was measured after 4 days of incubation with OVA. Data were obtained from six wells per group of mice. All results are representative of at least three independent experiments. ND, not detectable.

\section{Pravastatin treatment during OVA challenge suppresses antigen induced IL17 production of thoracic lymph node cells}

Pravastatin treatment during OVA challenge suppressed production of IL17 from immunocytes in the thoracic lymph nodes (fig 4). This result suggests that pravastatin suppresses the Th17 response in the thoracic lymph node as well.

\section{Pravastatin suppresses antigen presentation in the lung}

Finally, we examined the effect of pravastatin on antigen presentation in vivo. Treatment with pravastatin during the effector phase seemed to suppress the antigen presenting capacity of $\mathrm{CD} 11 \mathrm{c}^{+}$cells in the lung (fig 5). In our preliminary study, expression of major histocompatibility complex class II and co-stimulatory molecules such as CD40, CD80 and CD86 on $\mathrm{CD} 11 \mathrm{c}^{+}$cells from lung tissues did not differ between saline
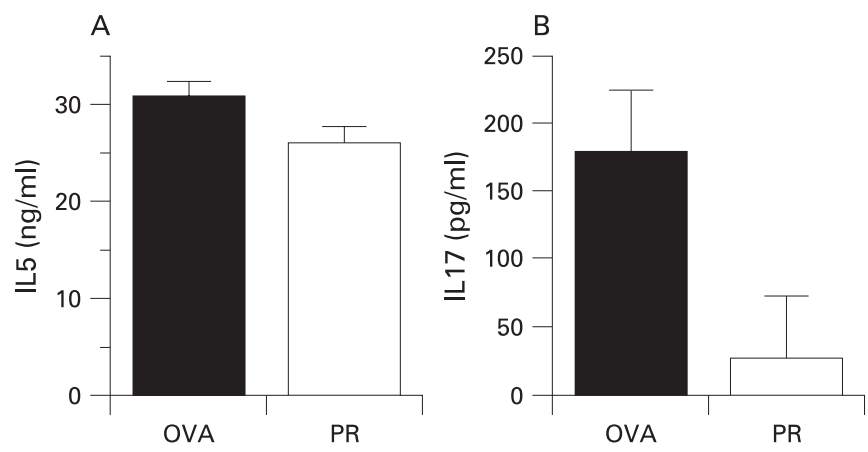

Figure 4 Treatment with pravastatin during ovalbumin (OVA) challenge attenuates antigen induced IL17 production of lymph node cells. (A, B) On day 21 , lymph node cells from OVA mice treated with pravastatin $(\mathrm{PR})$ or control vehicle (OVA) were incubated with OVA. After 3 days of incubation, production of IL5 (A) and IL17 (B) was measured. Data were obtained from six wells per group of mice. All results are representative of three independent experiments.

treated mice and OVA treated mice (data not shown). Therefore, we could not further assess the effect of pravastatin on expression of these molecules. In another experiment, we confirmed that treatment with pravastatin did not affect the migration of APCs (see supplementary fig 2 with method online). These results suggest that pravastatin suppressed the immune response in the airway partly by suppressing antigen presentation in the lung.

\section{DISCUSSION}

The results of the present study demonstrated that pravastatin suppresses sensitisation to OVA antigen with downregulation of IL17 production, and that it also attenuates the ongoing immune response in the airway. Pravastatin, delivered during systemic immunisation, attenuated the OVA induced Th2 type response with suppression of the production of IL17. In addition, pravastatin delivered during the airway challenge with OVA suppressed eosinophilic airway inflammation by affecting IL17 production, and suppressing the antigen presenting capacity of $\mathrm{CD} 11 \mathrm{c}^{+}$cells in the lung. As far as we know, this is the first study to clarify the effect of any statin treatment in vivo on IL17 production in an immune response and its effect on antigen presentation in the lung.

The immunomodulating effect of statins involves the inhibition of Th1-type responses. ${ }^{2}$ In addition, recent reports demonstrate that they can also suppress Th2-type responses. To date, two studies with simvastatin and one report with pravastatin demonstrated that statins suppressed the Th2-type allergic airway inflammation. ${ }^{10-12}$ In the current study, pravastatin tended to suppress OVA induced cell proliferation and cytokine production by spleen cells both ex vivo (fig 3) and in vitro (see supplementary fig 1 with method online). Pravastatin also suppressed eosinophilic airway inflammation, IgE production in serum and cytokine production from immunocytes in the thoracic lymph node (figs 1, 2, 4). These results are reasonably consistent with the findings of previous reports, ${ }^{10-12}$ confirming that statins certainly suppress Th2-type allergic airway inflammation in an experimental system.

To date, only one study has examined the effect of pravastatin on allergic airway inflammation. ${ }^{12}$ Yeh and Huang reported that treatment of mice with pravastatin from 2 weeks before antigen sensitisation to after an antigen challenge reduced airway eosinophilia. ${ }^{12}$ In a preliminary experiment, 


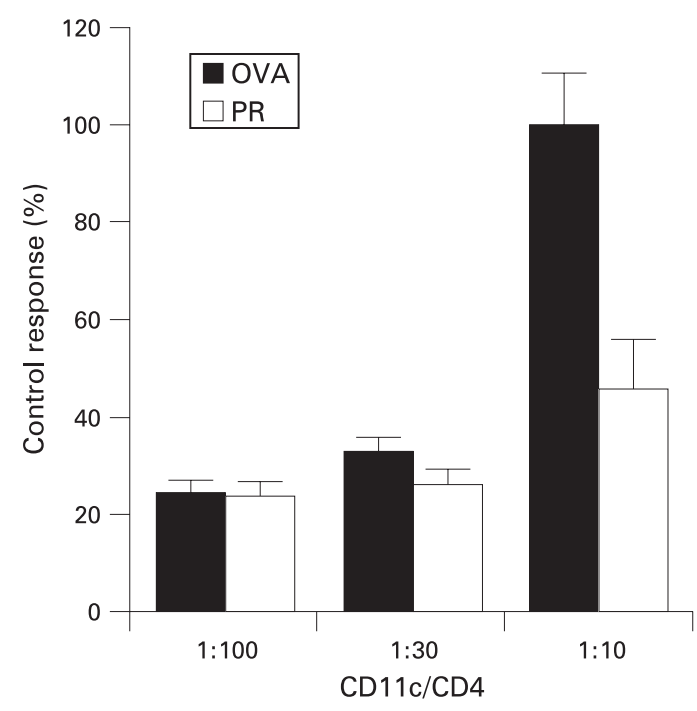

Figure 5 Treatment with pravastatin significantly suppressed the antigen presenting capacity of lung cells. On day $21, \mathrm{CD}_{11} \mathrm{c}^{+}$antigen presenting cells (APCs) in the lung of mice treated with pravastatin (PR) or saline (ovalbumin (OVA)) were positively selected and cocultured with $\mathrm{CD}^{+} \mathrm{T}$ cells from D011.10 mice. After 2 days of coculture, cell proliferation was measured. Data are expressed as a percentage of the response compared with that induced by APCs obtained from saline treated mice at an APC to $C D 4^{+} \mathrm{T}$ cell ratio of 1:10. Data were obtained from six wells per group. Results are representative of three independent experiments.

however, we could not reconfirm their results (data not shown). The reason for this discrepancy is not clear at present.

In this study, pravastatin tended to suppress OVA induced IL17 production in the spleen ex vivo and in vitro (fig 3 and supplementary fig 1 with method online). A recent study reported that simvastatin treatment in vitro directly inhibits IL17 production by inhibiting expression of IL17 transcription factor RORC in human $\mathrm{CD}^{+} \mathrm{T}$ cells. ${ }^{32}$ The role of IL17 producing Th17 cells in various immune responses has been highlighted recently. ${ }^{1427}$ The role of IL17 in asthma has been elucidated in humans, ${ }^{22}$ experiments in vitro ${ }^{26}{ }^{27}$ and animal models. ${ }^{2-25}$ IL17 induces cytokine/chemokine production by human bronchial fibroblasts ${ }^{26}$ and by human airway smooth muscle cells. ${ }^{27}$ These studies indicate that IL17 may enhance allergic reactions in the airway. In animal studies, OVA induced airway inflammation was reduced in IL17 deficient mice ${ }^{24}$ and in IL17 receptor deficient mice. ${ }^{23}$ In the current study, suppression of the OVA induced immune response by pravastatin was associated with suppression of IL17 production (fig 3 and supplementary fig 1 online). These findings support the idea that IL17 plays a critical role in antigen sensitisation.

In the effector phase of airway inflammation, lung IL17 content was increased in the OVA treated mice compared with the control (fig 1E). This is consistent with the findings of previous reports. ${ }^{23}{ }^{24}$ Two studies suggested that IL17 may play a protective role in the lung in the effector phase. ${ }^{23}{ }^{25}$ In contrast, in the present study, pravastatin suppressed IL17 production as well as Th2-type cytokine production by the lymph node (fig 4), and suppressed eosinophilic airway inflammation (fig 1). The role of IL17 or Th17 cells in the effector phase in the lung is not fully clarified at present, and further studies should be conducted.

Finally, to elucidate a novel role of statins in the lung immune response, we examined the effect of pravastatin on antigen presentation. Pravastatin tended to suppress the antigen presenting capacity of lung $\mathrm{CD}_{11 \mathrm{c}^{+}}$cells (fig 5). To date, the effect of statins on APC function has been elucidated mostly in vitro. ${ }^{3} 33{ }^{34}$ Only a few studies have reported the effect of statins on APCs in vivo. ${ }^{5}$ The effect of statins on APCs, especially in the lung, has not been examined, and the current study for the first time confirmed that pravastatin attenuates antigen presentation in the lung.

Our findings and previous studies with simvastatin ${ }^{10} 11$ indicate that statins could inhibit the progression of ongoing airway inflammation in patients with bronchial asthma. However, whether the results obtained with an animal model can be adapted to human asthma remains unclear. Although the animal model using ovalbumin is one of the most popular methods of analysing allergic airway inflammation, there are some differences between this model and human asthma. Firstly, ovalbumin is not a true antigen of asthma. Secondly, sensitisation by intraperitoneal injection of ovalbumin/alum is an artificial system. Furthermore, the dose of pravastatin is important in the clinical situation. In a preliminary study, treatment with $2 \mathrm{mg} / \mathrm{kg} /$ day of pravastatin did not attenuate allergic airway inflammation (data not shown). The dose of $10 \mathrm{mg} / \mathrm{kg} /$ day of pravastatin in this study is higher than the dose used clinically in human. The clinical dose of pravastatin in Japan is about $0.2-0.4 \mathrm{mg} / \mathrm{kg} /$ day, and the concentration in serum is $0.1-1 \mu \mathrm{M}$. On the other hand, the doses of statins used in most animal studies were higher than the clinical dose, and high doses of statins could suppress the activities of autoimmune disease, but did not show cholesterol lowering effects in mice. ${ }^{2}$

As animal models have limitations to adaptation to human diseases, clinical studies need to be performed. To date, only one clinical study has examined the effect of statins on chronic asthma. Menzies et al reported that simvastatin treatment for 1 month did not exhibit significant therapeutic anti-inflammatory effects. ${ }^{35}$ However, in their study, the number of patients enrolled in the trial was small, and simvastatin was administered during a stable period of asthma. To draw a definite conclusion, further clinical studies in various clinical situations are necessary.

In conclusion, we demonstrated that pravastatin suppresses eosinophilic airway inflammation by attenuating the sensitisation to allergens with suppression of IL17 production and by regulating lung dendritic cells in a murine model. Although further investigation is needed to confirm this possibility, our results indicate the therapeutic potential of statins as antiinflammatory drugs for asthma.

Acknowledgements: We thank Kanae Kurosaki for technical assistance. Competing interests: None.

Ethics approval: All experiments were approved by the Animal Research Ethics Board of the Department of Allergy and Rheumatology, University of Tokyo.

\section{REFERENCES}

1. Nakamura H, Arakawa K, Itakura H, et al. Primary prevention of cardiovascular disease with pravastatin in Japan (MEGA Study): a prospective randomised controlled trial. MEGA Study Group. Lancet 2006;368:1155-63.

2. Greenwood J, Steinman L, Zamvil SS. Statin therapy and autoimmune disease: from protein prenylation to immunomodulation. Nat Rev Immunol 2006;6:358-70.

3. Yilmaz A, Reiss C, Tantawi 0, et al. HMG-CoA reductase inhibitors suppress maturation of human dendritic cells: new implications for atherosclerosis. Atherosclerosis 2004;172:85-93.

4. Yilmaz A, Reiss C, Weng A, et al. Differential effects of statins on relevant functions of human monocyte-derived dendritic cells. J Leukocyte Biol 2006;79:529-38.

5. Youssef S, Stuve 0, Patarroyo JC, et al. The HMG-CoA reductase inhibitor, atorvastatin, promotes a Th2 bias and reverses paralysis in central nervous system autoimmune disease. Nature 2002;420:78-84. 
6. Lawman S, Mauri C, Jury EC, et al. Atorvastatin inhibits autoreactive B cell activation and delays lupus development in New Zealand black/white F1 mice. J Immunol 2004;173:7641-6.

7. Hakamada-Taguchi R, Uehara Y, Kuribayashi K, et al. Inhibition of hydroxymethylglutaryl-coenzyme A reductase reduces Th1 development and promotes Th2 development. Circ Res 2003;93:948-56.

8. Dunn SE, Youssef $S$, Goldstein MJ, et al. Isoprenoids determine Th1/Th2 fate in pathogenic $T$ cells, providing a mechanism of modulation of autoimmunity by atorvastatin. J Exp Med 2006;20:401-12.

9. Leung BP, Sattar N, Crilly A, et al. A novel anti-inflammatory role for simvastatin in inflammatory arthritis. J Immunol 2003;170:1524-30.

10. Mckay A, Leung BP, Mclnnes IB, et al. A novel anti-inflammatory role of simvastatin in a murine model of allergic asthma. J Immunol 2004;172:2903-8.

11. Kim DY, Ryu SY, Lim JE, et al. Anti-inflammatory mechanism of simvastatin in mouse allergic asthma model. Eur J Pharmacol 2007;557:76-86.

12. Yeh YF, Huang SL. Enhancing effect of dietary cholesterol and inhibitory effect of pravastatin on allergic pulmonary inflammation. J Biomed Sci 2004;11:599-606.

13. Hothersall E, McSharry C, Thomson NC. Potential therapeutic role for statins in respiratory disease. Thorax 2006;61:729-34.

14. Weaver CT, Hatton RD, Mangan PR, et al. IL-17 family cytokines and the expanding diversity of effector T cell lineages. Annu Rev Immunol 2007;25:821-52.

15. Harrington LE, Hatton RD, Mangan PR, et al. Interleukin 17-producing $\mathrm{CD}^{+}$effector T cells develop via a lineage distinct from the Thelper type 1 and 2 lineages. Nat Immunol 2005;6:1123-32.

16. Bettelli E, Carrier Y, Gao W, et al. Reciprocal developmental pathways for the generation of pathogenic effector $T_{H} 17$ and regulatory T cells. Nature 2006;441:235-8.

17. Happel KI, Dubin PJ, Zheng M, et al. Divergent roles of IL-23 and IL-12 in host defense against Klebsiella pneumoniae. J Exp Med 2005;202:761-9.

18. Khader SA, Bell GK, Pearl JE, et al. IL-23 and IL-17 in the establishment of protective pulmonary $\mathrm{CD}^{+} \mathrm{T}$ cell responses after vaccination and during Mycobacterium tuberculosis challenge. Nat Immunol 2007;8:369-77.

19. Cua DJ, Sherlock J, Chen Y, et al. Interleukin-23 rather than interleukin-12 is the critical cytokine for autoimmune inflammation of the brain. Nature 2003:421:744-8.

20. Murphy CA, Langrish CL, Chen Y, et al. Divergent pro- and antiinflammatory roles for IL-23 and IL-12 in joint autoimmune inflammation. J Exp Med 2003;198:1951-7.

21. Laan M, Cui ZH, Hoshino H, et al. Neutrophil recruitment by human IL-17 via C-X-C chemokine release in the airways. J Immunol 1999;162:2347-52.
22. Barczyk A, Pierzchala W, Sozanska E. Interleukin-17 in sputum correlates with airway hyperresponsiveness to methacholine. Respir Med 2003;97:726-33.

23. Schnyder-Candrian S, Togbe D, Couillin I, et al. Interleukin-17 is a negative regulator of established allergic asthma. J Exp Med 2006;203:2715-25.

24. Nakae S, Komiyama Y, Nambu A, et al. Antigen-specific T cell sensitization is impaired in IL-17-deficient mice, causing suppression of allergic cellular and humoral responses. Immunity 2002;17:375-87.

25. Hellings PW, Kasran A, Liu Z, et al. Interleukin-17 orchestrates the granulocyte influx into airways after allergen inhalation in a mouse model of allergic asthma. Am J Respir Cell Mol Biol 2003;28:42-50.

26. Molet S, Hamid Q, Davoine F, et al. IL-17 is increased in asthmatic airways and induces human bronchial fibroblasts to produce cytokines. J Allergy Clin Immunol 2001; 108:430-8.

27. Dragon S, Rahman MS, Yang J, et al. IL-17 enhances IL-1 $\beta$-mediated CXCL-8 release from human airway smooth muscle cells. Am J Physiol Lung Cell Mol Physiol 2007;292:L1023-9.

28. Okunishi K, Dohi M, Nakagome K, et al. A novel role of cysteinyl leukotrienes to promote dendritic cell activation in the antigen-induced immune responses in the lung. J Immunol 2004;173:6393-402.

29. Okunishi K, Dohi M, Nakagome K, et al. A novel role of hepatocyte growth factor as an immune regulator through suppressing dendritic cell function. J Immunol 2005; 175:4745-53.

30. Nakagome K, Dohi M, Okunishi K, et al. In vivo IL-10 gene delivery suppresses airway eosinophilia and hyperreactivity by down-regulating APC functions and migration without impairing the antigen-specific systemic immune response in a mouse model of allergic airway inflammation. J Immunol 2005:174:6955-66.

31. Brandt EB, Strait RT, Hershko D, et al. Mast cells are required for experimental oral allergen-induced diarrhea. J Clin Invest 2003;112:1666-77.

32. Zhang $\mathbf{X}$, Jin J, Peng $\mathbf{X}$, et al. Simvastatin inhibits IL-17 secretion by targeting multiple IL-17-regulatory cytokines and by inhibiting the expression of IL-17 transcription factor RORC in CD4+ lymphocytes. J Immunol 2008;180:6988-96.

33. Pahan K, Sheikh FG, Namboodiri AM, et al. Lovastatin and phenylacetate inhibit the induction of nitric oxide synthase and cytokines in rat primary astrocytes, microglia, and macrophages. J Clin Invest 1997:100:2671-9.

34. Kwak B, Mulhaupt F, Myit S, et al. Statins as a newly recognized type of immunomodulator. Nat Med 2000;6:1399-402.

35. Menzies D, Nair A, Meldrum KT, et al. Simvastatin does not exhibit therapeutic antiinflammatory effects in asthma. J Allergy Clin Immunol 2007;119:328-35. 